\title{
Déversement du regard fluide: esquisse d'une méthodologie pour approcher théoriquement le cinéma
}

\author{
Molina-García, Érika Natalia
}

Université Toulouse II Jean Jaurès - Université Charles de Prague, aruthmos@gmail.com

\begin{abstract}
Resumen
En su obra de 2010, Éric Thouvenel analiza la profusión de imágenes liquidas en un periodo preciso del cine francés, interpretándola como un síntoma, no solamente de un tiempo turbulento como fue el transcurrido entre las dos guerras mundiales, sino que del tiempo en sí mismo. En efecto, a comienzos del siglo XX el desarrollo de las tecnologías cinematográficas y el fin del cine mudo se vieron acompañados por un redescubrimiento del conjunto de la realidad como líquida, y en el mismo movimiento de un reposicionamiento de las cuestiones de la temporalidad y el devenir al centro de todas las áreas del pensamiento. Un umbral es así atravesado, desde un paradigma simbólico telúrico a uno acuoso. Este pasaje justifica suficientemente la obra de Thouvenel que busca penetrar el cine con una mirada en sí misma líquida, pero justifica igualmente una sistematización de dicha mirada, y su aplicación a un caso concreto como es el cine de Philippe Garrel.
\end{abstract}

Palabras clave : Éric Thouvenel ; Philippe Garrel ; Gilles Deleuze ; mirada fluida ; filosofía del cine.

\section{Résumé}

Dans son ouvrage de 2010 (Les images de l'eau dans le cinéma français des années 20), Éric Thouvenel interroge la profusion des images de la liquidité dans une période bien précise du cinéma français, tout en l'interprétant comme un symptôme du temps ; non seulement de l'époque trouble de l'entre-deux-guerres, mais du temps en tant que tel. En effet, le développement des technologies cinématographiques et la fin du cinéma muet s'accompagne d'une redécouverte de l'ensemble de la réalité comme liquide, et ainsi d'une remise du dynamisme, de la temporalité et du devenir, au centre de la pensée. On traverse ainsi un seuil, engageant un changement de paradigme symbolique, d'un modèle tellurien vers un modèle aquatique. Ceci justifie non seulement la démarche de Thouvenel qui s'emploie à analyser le cinéma d'un regard lui-même fluide, mais également une systématisation de ce regard pour l'appliquer au cinéma de Philippe Garrel.

Mots-clés : Éric Thouvenel ; Philippe Garrel ; Gilles Deleuze ; regard fluide ; Philosophie du cinéma.

\begin{abstract}
In his book from 2010, Eric Thouvenel analyses the profusion of liquidity images in a specific period of French cinema, by interpreting it as a symptom of times; not only of a troubled era, but of Time itself. In the early twentieth century the development of film technology and the end of silent movies is indeed accompanied by a rediscovery of the whole of reality as fluid. This puts again the questions of temporality and becoming at the center in all areas. We seem in this manner to undertake a change of symbolic paradigm, leaving a tellurian model to immerse ourselves in a liquid one. This justifies Thouvenel's work, wanting to analyze cinema with a look itself fluid, but also the systematization of this fluid look and its application to Philippe Garrel's cinema.
\end{abstract}

Keywords : Éric Thouvenel ; Philippe Garrel ; Gilles Deleuze ; fluid look ; Film philosophy. 


\section{Introduction}

Depuis au moins deux siècles on assiste à un devenir liquide progressif de notre regard sur le monde, la réalité mettant en question nos anciens dogmatismes telluriens et nous contraignant à une souplesse et un dynamisme aquatiques. Les témoignages d'un tel changement de paradigme symbolique, comme l'ouvrage collectif paru en 2013 sous le titre L'Impressionnisme, les arts, la fluidité, sont nombreux, et il nous semble important de les analyser. C'est ainsi que nous allons nous concentrer sur l'ouvrage Les images de l'eau dans le cinéma français des années 20 d'Éric Thouvenel, paru en 2010. Il s'agira de mettre en valeur l'effort entrepris par l'auteur à penser le cinéma comme art d'immersion qui convoque tous nos sens lorsque nous pénétrons dans le noir de la salle.

De la traversée de cette véritable sculpture aquatique que Thouvenel bâtit en 9 chapitres, répertoriant, analysant et interprétant 72 films français de la décennie de l'entre-deux-guerres, évoquant comment opère la mutation liquide du paradigme symbolique dans des champs aussi divers que celui du social, de l'art, de la philosophie (notamment grâce à la pensée bergsonienne) et de la science (avec la mécanique des fluides) - de cette traversée donc, nous allons distiller une méthode, une sonde théorique au moyen de laquelle forer les films, en l’occurrence ceux du réalisateur Philippe Garrel, né en 1948 et très actif encore dans la scène filmique française.

Mais avant de commencer, on peut demander à Thouvenel quels sont les motifs de son choix. Pourquoi a-t-il préféré cet espace-temps précis qu'est le registre filmique de la France des années 20, afin d'entamer une recherche sur un motif aussi universel que celui de l'eau. Et la réponse sera que précisément la présence et la valeur symbolique de l'eau au cinéma étant tout simplement massive, exige bien un choix, une délimitation du terrain d'analyse, et ensuite que cette période de l'après-guerre est celle qui voit la fin de la gestation du cinéma -le cinéma muet-, donnant lieu au septième art en tant que tel, avec des voix et des bruitages, c'est-à-dire du cinéma actuel. Cette période de gestation du cinéma comme art, en France a été vécue largement comme une expérience hydroponique.

Finalement, ce que l'école française trouvait dans l'eau, c'était la promesse ou l'indication d'un autre état de perception : une perception plus qu'humaine, une perception qui n'était plus taillée sur les solides, qui n’avait plus le solide pour objet, pour condition, pour milieu. Une perception plus fine et plus vaste, une perception moléculaire, propre à un “ciné-œil” (Deleuze, 1983 : 115, 116).

\section{Première partie: Epistémologie des fluides}

Reprenant le titre d'épistémologie des fluides formulé par Thouvenel en page 234, nous tentons d'exsuder une méthodologie simplement : observant pas à pas ce qui a été fait par l'auteur. Dans son travail il présente 3 sections que nous pouvons dénommer Territoire, Qualité et Parole. Dans cette méthode à trois vitesses il s'agit dans un premier temps de situer géographiquement, et dans le monde réel et dans la scène artistique, les images filmiques de l'eau ; dans un deuxième temps, de faire une analyse qualitative des rôles et fonctions de cet élément ; et finalement de repérer les images aquatiques dans la parole de ceux qui font du cinéma, dans une sorte de métalangage littéraire des réalisateurs à partir de la langue déjà émise dans leurs corpus d'images-mouvement. Territoire, Qualité et Parole : voilà la structure de la méthode qu'il nous faut suivre.

La première partie de l'ouvrage « Les territoires du cinéma : Une histoire des représentations de l'eau comme Paysage », consiste en trois chapitres et montre comment l'auteur, loin de se livrer à une analyse purement esthétique, explore les explications historiques et sociologiques de la profusion des images de l'eau dans les films. La crise de l'après-guerre et le succès du style américain au cinéma, auraient ainsi été des facteurs déterminants pour imprimer dans les pellicules les éléments aquatiques. En effet, le cinéma de l’eau en milieu naturel aurait été à la fois une réponse économiquement bien adaptée, car filmer en extérieur devenait clairement plus accessible, et une réponse identitaire, lorsque cette sortie du studio permettait une découverte du paysage français par l'écran. L’eau commence alors à jaillir dans les salles de cinéma grâce à l'élan d'auto-préservation du cinéma français. Mais Thouvenel amène cette analyse du contexte social bien plus loin, lorsqu'il rapporte les anecdotes de l'émergence concrète des grands studios Pathé puis Gaumont à Nice (1910-), et leurs rapports avec les cinéastes d'avant-garde. Ces artistes auraient bien profité des commandes à tendance commerciale pour développer leur modernité. Ce genre de narrations en guise d'explication, nous permet de voir comment aux yeux 
de Thouvenel aucun détail, quoiqu'il semble accessoire, ne doit être laissé de côté par celui qui cherche la cause d'un trait du cinéma, tel le déluge d’images aquatiques dans les années 20.

Contextualiser les forces en présence est en effet indispensable si l'on cherche à mieux comprendre comment cet ensemble de choix stratégiques a pu favoriser à la fois la multiplication des tournages en bord de mer ou de rivière et l'apparition d'une cinématographie consciente de sa portée théorique, engagée dans une posture d'autojustification et pour laquelle la fluidité de l'élément aquatique, sa propension à accueillir les multiples configurations de lumière, seront décisifs dans ce qui fut aussi une bataille pour la reconnaissance (Thouvenel, $2010: 26$ ).

De cette façon, ce à quoi nous assistons avec ce livre c'est à une inondation de la scène sociale par ce regard fluide : toute dimension et tout facteurs peut et doit être exploré. Thouvenel va même jusqu’à analyser le tourisme, l'expansion des routes ou les innovations techniques et leur rapport au cinéma en tant qu'industrie, pour rendre compte des images de l'eau. Ainsi nous pourrons résumer cette mer profonde de causalités entrecroisées, en trois aspects : individuel (le cinéaste en quête de devenir artiste, de la définition et la réalisation du septième art), identitaire (l'Etat et l'industrie qui cherchent à exalter le sentiment de patriotisme) et urbain, avec des citoyens souffrant de plus en plus de la migration urbaine et de leur enfermement dans les grands villes, ayant besoin de l'air et de l’eau dans l’échappé qui procure ce cinéma, « dont les vertus descriptives et l’impact sur l’imaginaire collectif ne sont plus à démontrer » (Thouvenel, 2010 : 31).

Tout en construisant une cartographie, non pas homogène, mais en soulignant les hauts lieux susceptibles d’être repérés, Thouvenel prolonge cette partie par une analyse des genres de film français à tendance aquatique parus dans cette période, et dégage ainsi 4 catégories, correspondant chacune à des personnages et des scenarios assez déterminés, ainsi qu’à des rapports spécifiques à des expressions corrélatives dans les champs de la littérature et la peinture.

Les genres du film aquatique seront déterminés grâce aux cinq grands lieux de la cartographie aquatique, à savoir, premièrement, les cours d'eau -fleuves, canaux, ruisseaux, système sanguin et nerveux du territoire (Éric Thouvenel, 2010 : 45) ; deuxièmement, le port et l'expérience du voyage ; troisièmement, le rivage et l'expérience des limites ; quatrièmement, l'île, le phare et les territoires indécis ; et en dernier terme, le jet d'eau et la synthèse symbolique qu'il opère au cœur de la transition mécano-philosophique de tout cinéma.

Ainsi, les films des années 20 pourraient être rangés dans une première catégorie naturaliste ou idéaliste (films de péniche, à penchant bucolique) ; une deuxième dénommée Marines, empruntant le nom à l'éponyme pictural et se déroulant évidemment dans la mer ou au bord de mer, dans son immensité violente et conflictuelle ; une troisième catégorie, prenant comme sujet et décor la montagne, ses eaux aussi bien vives que solides; et une quatrième catégorie d'avant-garde, où les cinéastes sont touchés par le paradoxe universel de la théorie et la praxis, du concret et l'abstrait, cherchant d'un côté à dénoncer leur société grâce à des images d'eaux troubles ou fluviales, évoquant le changement, et d'un autre côté, cherchant à utiliser le cinéma comme heuristique philosophique, par l'utilisation d'une récurrence extrêmement variée des flaques, brume, remous, écume, jets, reflets, gouttes, vagues, et de toute substance aquatique au service d'un décryptage du monde.

Ces quatre catégories seront ainsi des réverbérations de ce que Thouvenel continue à développer jusqu’à la fin de cette première partie : la modernisation esthétique, voire la variation moderne de la perception qui abandonne grâce au cinéma une vision picturale bien plus statique du monde, et avance vers un dynamisme hydraulique. Cet abandon entamé déjà avant les années 20, se serait développée avec une force prodigieuse pendant les dernières années du muet, notamment par la mutation dans l'idée de paysage, à laquelle l'auteur dédie la dernière partie du premier chapitre.

Le mot paysage, apparu en langue française au cours du XVI ${ }^{\mathrm{e}}$ siècle, renvoie à l'invention d'une vision, non pas du monde tel quel, mais d'une mise en scène contrôlée de ses éléments, et serait un concept proprement pictural. Mais dès qu'avec le cinéma nous avons eu la possibilité de capturer le mouvement dans le temps, de varier les points de vue par le cadrage, le montage ou les mouvements de l'appareil filmique, nous avons eu accès à un tout autre degré d'invention, et ainsi à un tout autre concept de paysage, non pas contrôlant seulement l'espace, la lumière et la disposition des objets, mais aussi les rythmes, vitesses, profondeurs, et quasiment tous les éléments qui font d'une perception une perception vive. Processus de perfection de la perception filmique qui continue de nos jours avec les outils de réalité virtuelle. 
Pour Thouvenel la question du cinéma en général réside dans ce rapport perceptif au monde réel, et il questionne tout au long du livre la base de la croyance à la dichotomie réel/idéal. C’est ainsi qu'il se demande à la page 84 comment continuer à inventer des paysages, à inventer le monde pas encore existant avec le cinéma, c'est-à-dire, avec un dispositif de représentation si proche de la réalité. Et sa réponse sera notamment liée à l'eau.

[Les caractéristiques de l'eau] font en effet l'instrument idéal d'un va-et-vient constant entre la forme et l'informe, le réalisme et l'abstraction [...] il faut alors se demander comment l'ensemble des variations plastiques mises en jeu par les images de l'eau permet de donner corps à un paysage spécifiquement cinématographique [...] (mais) -continue l'auteur- Ces images qui ne cessent de transformer, d'hésiter entre le réel et son envers, peut-on dire encore que ce sont des "paysages" ? Assurément, si on les rapporte à l'acception picturale du terme, puisqu'il s'agit bien de mettre en forme la nature, d'en proposer une interprétation par la mise en place des cadres de vision qui sont à la fois matériels l'opération du cadrage-, plastiques et conceptuels. Et oui encore si l’on considère le paysage, au sens plus général du terme, comme une vue découpée par le regard (Thouvenel, 2010 : 89)

L'eau faciliterait ainsi particulièrement aux cinéastes la tache classique de l'art, à savoir : répondre à la double contrainte de représentation fidèle et d'innovation ou transformation plurivoque.

Dans la deuxième partie du livre, ayant pour titre À l'épreuve des images : statut et fonctions du motif aquatique, l'auteur s'occupera de deux questions (Chapitres 4 et 5) : d'une part, du dépassement qu’opère le motif aquatique de la vision dichotomique de l'art que nous venons d'évoquer (l'art soit comme reproduction de la réalité, soit comme art fictif, révolutionnaire), et d'autre part, de la question de savoir comment les propriétés optiques de l'eau sont au service d'une quête de ce qu'on peut appeler le visuel cinématographique.

Pour la première question Thouvenel montrera comment dans les années 20 le motif aquatique exprime une transformation du rôle des images des éléments naturels en général, passant du statut de simples décors à celui de véritables personnages, et comment ce motif indique une démultiplication des rapports au réel, plutôt que d'en faire une narration déterminée, signalant que :

[Quand on montre l'eau] placide ou furieuse, opaque ou limpide, mobile ou statique, dans et par le mouvement des images, on la donne à voir comme un acte à la fois narratif, figuratif et épistémologique. [...] [il s'agit d'une] volonté de faire concourir la nature au geste cinématographique, d'en dépasser la pure et simple saisie (Thouvenel, $2010: 113$ ) [...].

que les cinéastes l'envisagent comme personnage, support d'expression plastique ou cadre cinématographique à recomposer/atomiser dans l'espace du studio, c'est toujours la multiplicité de ses caractéristiques que les films exposent et valorisent (Thouvenel, $2010: 148$ ).

[...] La fiction est aussi ce qui permet au réel de s’affirmer autrement (Thouvenel, 2010 : 127).

Pour ce qui est du deuxième point (c'est-à-dire : comment les propriétés optiques de l'eau servent à vitaliser une mouvance non mimétique et antinarrative de l'art contemporain) Thouvenel cadre l'analyse de ces propriétés dans un large geste conceptuel, à savoir, le renversement de la logique aristotélicienne qui privilégia l'aspect mythologique ou l'intrigue de l'art sur l'opsis, sur son effet tangible sur le public. Il s'agit ainsi d'un changement du régime rationnel au régime sensible, des histoires aux affects, auquel contribuent les images de l'eau grâce à leurs propriétés de réflexion de la lumière, devenant eau-miroir, eau-écran et eau-tombe ; grâce à leurs modalités opaques et transparents, colorées après-coup ou en noir et blanc.

Enfin, Thouvenel traite dans la troisième et dernière partie de son ouvrage ( $L$ 'eau et la figuration du mouvement, ou comment Penser en mots et en images les puissances du cinéma comme art visuel -qui continue à développer l'importance de l'eau dans la constitution de l'art cinématographique) de quatre questions (chapitres 6, 7, 8 et 9) : premièrement, de la place de l'eau dans les théories cinématographiques des années 20 ; deuxièmement, du rapport de l'eau à la question du 
rythme dans ce contexte ; troisièmement, de quelques mots importants du langage ou lexique cinématographique ; et quatrièmement, de ce que Thouvenel appelle en reprenant le terme de Deleuze, une perception liquide.

Pour ce qui est de la première question, à savoir la fonction de l'eau dans les théories sur le cinéma, l'auteur aura l'occasion d'argumenter en faveur de l'affinité profonde qu’il perçoit entre l'élément liquide et le cinéma lui-même, grâce à la fréquence extrême de la métaphore aquatique dans les thématisations écrites sur le cinéma.

Les ondulations, les reflets, l'accéléré et le ralenti, les gros plans sur les vagues, la course des nuages, les plans en négatif, les surimpressions, le ruissellement, les écrans de brouillard, les miroitements, les jets, la buée, tous les avatars de l'eau deviennent la matière sensible à partir de laquelle les cinéastes déploient les ressources de leur moyen d'expression (Thouvenel, $2010: 194$ )

Thouvenel esquisse ainsi un inventaire du foisonnement des images de l'eau dans la critique du cinéma, approfondissant notamment deux lignes discursives, celle de la photogénie (selon la notion d’Epstein, c'est-à-dire considérant la photogénie comme la majoration morale d’une chose mobile dont la personnalité est révélée par le cinéma) et celle du cinéma pur, comme doctrine expérimentale du visuel qui à l'époque radicalisa l'avancée non langagière, anti-scénariste et militante, en proposant une optique puriste (évoquée plus haut, . Thouvenel, 2010 : 193).

[...] L’accéléré, le ralenti, la réversibilité du temps filmique et "l’échange des substances et des propriétés” sont les axiomes à partir desquels Epstein construit sa lente et rigoureuse démonstration, centrée autour d'une idée fixe : affirmer la primauté du devenir sur la permanence, et faire de la variabilité, de la fluidité, les points d'appuis d'une conception du monde que le cinéma éclaire et renouvelle (Thouvenel, 2010 : 201)

L'auteur finira ce point sur l'eau dans le discours des cinéastes autour du cinéma avec un recensement de l'aspect proprement philosophique du cinéma d’Epstein, comme figure majeure de la quête hydroponique du 7ème art, de cet art en devenir, se légitimant comme tel.

[...] c’est bien pourquoi les images de l'eau sont si présentes dans tout l'œuvre d’Epstein. Plus que la métaphore d'un système, l'élément figure le calque direct de sa pensée, dont il emprunte les formes, les états et la structure, autant qu’il y recourt à titre d'exemple. Epstein ne se contente donc pas d'affirmer "la nature foncièrement colloïdale de la matière” : c’est sa pensée elle-même qui adopte ce principe (Thouvenel, 2010 : 210)

Pour ce qui est de la deuxième question de cette dernière partie, le rythme, Thouvenel abondera dans l'élaboration de tautologies et leur expression en pléonasmes. Revenant à l'étymologie du terme rythme, signifiant littéralement « couler » (Thouvenel, 2010 : 212), évoquant l’idée d’un flux continu, les arts rythmiques en général, musique, danse et cinéma, devront chercher à définir leur autonomie, étant tous des mises en scène du temps, du mouvement lui-même. L'auteur nous rapportera ainsi à l'ancienne définition d’Abel Gance du cinéma comme musique de la lumière (Thouvenel, 2010 : 214) et analysera l'importance des images de l'eau dans la dichotomie rythmique du cinéma, entre les rythmes intérieurs du plan et les rythmes extérieurs du montage.

[...] tout film est un flux dont le contenu définit les reliefs, marque les accents [...] Quand nous regardons des plans d'une extrême simplicité, comme ceux des bouillonnements de l'eau dans un caniveau, de nuages qui traversent le cadre à des vitesses variables, d'une cascade ou d'un torrent, des clapotis de la mer contre un quai, du ressac de l'océan sur une grève ou de remous dans le sillage d'un bateau, nous ne voyons finalement rien d'autre que des mouvements en travail [...] "le temps apparu”. Et quand, à quelques années de distance, Abel Gance isole en gros plan l’image d'une stalactite qui goutte dans La Roue, et Joris Ivens celle de gouttes blanches qui tombent au sol à intervalles réguliers dans Pluie, l'un et l'autre ne font rien d'autre que mettre en scène, eux aussi, cette comparution du temps (Thouvenel, $2010: 221)$ 
Pour ce qui est de la troisième question, Thouvenel s'appliquera à chercher dans certains termes du langage cinématographique les traces de ce que les cinéastes ont pu apprendre grâce aux images de l'eau en termes de technique et de création artistique concrètes, voire non pas une réflexion théorique sur l'eau et le devenir, mais une « mise en signes du monde » (Thouvenel, 2010 : 240) du point de vue du développement technique que les avatars de l'eau ont exigé pour se donner à imprimer en pellicule. Ainsi Thouvenel finit sa décomposition de la perception liquide en tant que telle, analysant certains avatars de l'eau, notamment les nuages, la brume et la neige, ainsi que certaines figures cinématographiques comme le flou, l’arrêt sur image, le gel d’images et certains mélanges d’images, sans manquer en analyses des surimpressions et des fondus enchainés (Thouvenel, $2010: 273$ ).

\section{Deuxième partie: Regard extravasé}

Nous nous proposons maintenant d'étendre le point de vue de Thouvenel aux films de Philippe Garrel, et souhaitons identifier les territoires, les qualités et les paroles qui tourbillonnent autour de l'eau présente dans ses films. Pour territorialiser et contextualiser l'œuvre de Garrel nous devons rappeler qu'il s’agit d'un travail très intuitif, réalisé dès un très jeune âge avec Les enfants désaccordés en 1964, quand Philippe Garrel ne compte que 16 ans, jusqu’à nos jours. Ce travail sera largement imprégné des évènements de mai 1968, qui reviendront non seulement représentés dans certaines scènes, comme dans Les amants réguliers de 2005, mais aussi et surtout dans sa disposition de réalisateur. En effet, dans ses conférences les plus récentes on observe un Garrel extrêmement conscient de que ses spectateurs sont des bourgeois, des gens disposant d'assez de temps et d'argent pour aller dans les salles de projection. Il s'agit encore d'un réalisateur qui, tout en étant assez restrictif du point de vue des analyses théoriques, rapidement jugées prétentieuses, ira jusqu’à penser les films d'amour et désamour, de couples et d'individus - Nouvelle vague et autres - comme une action de résistance contre ce qu'il appelle le cinéma de Pétain (Philippe Garrel, 2014) : cinéma grandiloquent de l'élitisme et la patrie. Ses films seront ainsi ceux des blessures à portée de peau, à taille humaine et telles qu'elles se manifestent dans un croissement de regards. Dans ce sens les images de l'eau auront dans ses films souvent une présence ordinaire, celle du quotidien, mais décisive par leur qualité expressive.

Nous avons l'impression de voir se développer chez Garrel, de plus en plus et en sourdine les idéaux des années soixante qui l'ont habité dans sa jeunesse, jusqu'à arriver à un réalisateur qui redécouvre le cinéma non seulement comme art à part entière et subjectif, impuissant à s’imposer à personne comme valeur de vérité, mais aussi comme art procédant de la subjectivité de chacun de ses personnages, et non seulement du protagoniste principal. C’est ainsi une déhiérarchisation opérant à tous les niveaux du $7^{\text {ème }}$ art qui voit le jour grâce au cinéma de Philippe Garrel.

Or grâce à l'application de la méthode de Thouvenel nous pouvons apprécier que dans le cinéma de Garrel les avatars concrets de l'eau se concentrent sur la plage et la mer, les quais de Seine et les ponts où se retrouvent les amoureux ou ceux qui sont prêts à se suicider. Nous trouverons aussi souvent de la pluie, l'eau sur la table, celle de tous les jours, transparente et calme, mais aussi dans le court métrage de 1984 Rue Fontaine l'on observera de l'eau dans un rôle mortifère : eau ciguë, empoisonnée. On retrouve aussi fréquemment l'eau des baignoires, mais sans oublier que ce sont des bains de personnages qui ne parviennent jamais dans les films de Garrel à nettoyer leurs soucis ou leurs folies. Finalement, il faut porter notre attention sur un certain conflit entre deux avatars de l'eau à portée presque cosmologique, qui -au-delà du bien et du mal- vont être constamment en conflit dans les premiers films de Garrel : il s'agit du conflit entre le liquide amniotique, l'eau vive des ventres, l'eau intérieure de la grossesse des femmes, des enfants qui dorment et se développent, et les eaux démesurées de la mer, pure extériorité, eaux chaotiques d'infinies promesses ou d'un vide engloutissant. Entre ces deux sortes de liquidité, la liquéfaction de la mer chaotique va toujours gagner la bataille.

Ce dernier cas de figure nous permet d'aborder l'aspect qualitatif des eaux dans ces films, puisqu'il montre que même si les apparitions liquides jouent souvent des rôles contradictoires, c'est toujours une seule version du bleu des origines qui s’impose : l’immensité de la mer. De là la structure du film de 1979 Le bleu des origines : c'est le bleu de la pellicule, dépourvu de toute humanité, celui duquel ce film -et le grand tournage qui est l'existence même- va commencer et auquel il retournera.

Non pas une eau maternelle ou féminine, mais l'infini des possibles et des impossibles, inqualifiable, sans connotation positive ni négative, hors du binarisme sexuel. Ce bleu des origines est toujours le bleu de la machine ou de la nature. 
C'est pourquoi, lorsqu'on tente de s'échapper sur terre et non pas vers la mer chaotique, on ne peut que tourner en rond. Il est nécessaire que l'évasion soit faite dans l'élément liquide. C'est ce qui arrive dans Le lit de la vierge, tourné en 1969 dans la baie des Trépassés en Finistère, quand le comédien Pierre Clémenti laisse doucement la vierge enceinte sur le sable et s’interne dans les vagues. En contraste, Garrel tente en vain de s’échapper par terre dans La cicatrice intérieure (premier film avec Nico des Velvet underground, tourné entre 1970 et 1972) où le couple nous livre une des scènes les plus sublimes du cinéma : dans le désert du Nouveau-Mexique, sur fond de la voix de Nico qui chante Janitor of lunacy, priant à l’huissier de la folie qu’il la garde de la déraison, tandis que son corps crie et brimbale, vaincu.

Nico tient la main de Garrel. Il regarde ailleurs, nulle part. Sans enthousiasme mais avec persistance et force, Garrel arrache sa main de celle de Nico et entame une marche totalement épuisée et sans attente. Ses pieds se lèvent à peine et sans s'en rendre compte il accomplit l'étonnant tour complet d'un chemin qui semblait indéterminé, mais qui ne reconduit qu'à nouveau face à ce qu'il venait de quitter. Cercle symbolique de l'enfermement tellurique, anhédonie : Garrel revient sans avoir avancé à Nico, qui continue à se convulser. Sa tête doit se pencher violement pour ramener son regard de ce nulle part au monde des faits où après une simple boucle il retrouve la même femme pleurnichante. Il fallait partir dans l'eau pour partir vraiment.

Pour finir et concernant le troisième point de la méthode (la parole), il faut souligner que Garrel rejoint tout à fait, par son discours, la lutte de reconnaissance du cinéma comme septième art que Thouvenel avait identifié comme fond des motivations de la présence des images de l’eau dans le cinéma des années 20. Avec Garrel le cinéma continue à s'autofonder ; ses films n’étant rien de moins que l’incarnation de tous les débats théoriques analysés par Thouvenel qui opposent le réel et le fictif, l'authentique et le faux. Non seulement derrière, mais souvent devant la caméra, Garrel accomplit un cinéma autobiographique, où la notion d’auto- renvoi à la fois à sa propre subjectivité, au cinéma lui-même, à chaque personnage et chaque comédien, souvent conviés à improviser leurs dialogues.

Sur ce fait est surtout parlante la comparaison qui revient sans cesse dans la bouche du réalisateur avec la peinture, car elle n'est pas là seulement pour justifier le statut artistique du cinéma et légitimer sa propre démarche, ni non plus seulement pour faire voir comment il travaille avec des matières aussi liquides et déprédictives que l'huile (le jeu des comédiens), mais surtout pour revendiquer le passage d'un régime rationaliste à un régime sensible si cher aux premiers avant-gardistes.

Quand je crée avec une caméra j'ai vraiment l'impression que je ne peux pas faire autrement par rapport à ce que j'ai devant les yeux et la scène que je fais, donc je n’ai pas de théorie sur ce qui doit être le résultat du film [...] c’est comme la peinture, ce n'est pas de l'hyper-lucidité. [...] C'est gestuel, ce n’est pas forcement intellectuel. [...] C’est un habilité des sens. [...] Ce n'est pas forcement quelque chose qui naît de l'intelligence [...] Parce que c'est un art, ce n'est pas juste le résultat d'un savoir ou d'un travail. (Garrel, 2014 : min. 34)

\section{Conclusion}

Les images de l'eau dans le cinéma sont un élément fondamental aussi bien au niveau expressif que d'autolégitimation de cette forme d'art. L'ubiquité et la variété de ces images exige une méthodologie relativement stricte et exhaustive, comme celle que nous avons présentée grâce à l'ouvrage d’Éric Thouvenel. L'application de ce regard aux films de Garrel -regard fluide autant par son objet thématique que par sa disposition à couler dans les divers registres qui composent un objet cinématographique- requiert un travail bien plus long que celui que nous avons pu accomplir dans ces quelques lignes, où nous avons pu évoquer juste quelques avatars aquatiques importants. Une analyse beaucoup plus longue des techniques cinématographiques liées à l'élément liquide et leurs rapports aux archétypes optiques de l'eau dans les films tels qu’ils ont été traités par Thouvenel (eau-miroir, eau-écran et eau-tombe), s’impose.

Pareillement, il faut approfondir les philosophies par lesquelles cette traversée nous a fait passer, car rendre notre méthode et notre œil liquides signifie de lui permettre d'exprimer sa nature d’humeur aqueuse attrapé dans des membranes, c'està-dire lui permettre d'interpréter les images de l'eau au-delà des structures et binarismes théoriques. Ce n'est que l'eau qui consentira un tel développement, car les éléments comme la terre, l'air et le feu, sont tendus entre la vie et la mort, et donc entre des connotations négatives et positives, alors que seule l'eau, entre tous les éléments dynamiques - entre tous les éléments qui ne sont pas telluriques donc - expose le chaos non-humain d'infinités perçu aussi bien par un Deleuze 
que par un Garrel. D’un point de vue symbolique, l'air et le feu sont encore des éléments actifs qui relèvent, selon une certaine lecture philosophique, d’un humanisme phallique qui hiérarchise et préfère l'activité à la passivité. Seule l'eau garantie dans ce sens une issue, seule l'eau qui n’est ni féminine ni continente, ni masculine ni fécondante ; ni vertige qui effraie, ni mystère qui attire ; ni «l'eau verte » (Arthur Rimbaud, 2001 : 40), ni l'eau claire comme les «larmes d'enfance » (Arthur Rimbaud, 2001 : 60) ; seule l'eau, qui n'est rien de cela et tout à la fois, car elle reste un abyme insondable d'infinies virtualités.

Deux ruisseaux de recherche restent ainsi ouverts : d'un côté, celui des images de l'eau dans le cinéma, notamment chez Garrel, ses miroirs et ses fantômes liquides imprimés en pellicule, ses rythmes et sa plastique, et d'un autre côté, celui des philosophies adaptées à ce nouveaux paradigme aquatique non-binaire. Ces philosophies sans source et sans écluses, pourrons nous aider à explorer le cinéma, symétriquement à l'exploration que le cinéma peut faire des divers paradigmes de pensée. N’oublions pas qu’il existe une fraternité profonde entre cinéma et philosophie, non seulement car les deux confluents dans leur souci commun pour thématiser le mouvement, mais parce que les deux portent au cœur de leur sens une quête radicale de justesse et justice. Le cinéma n’étant rien de moins que la guerre contre les images fausses dans une compossibilité infinie de toutes les formes d'art (Badiou, 2014).

\section{Références bibliographiques}

BAdiou, Alain (2014). Cinema and Philosophy. Sydney : Université de Nouvelle-Galles du Sud, Faculté d’Arts et sciences sociales. $<$ https://www.youtube.com/watch?v=Arwso3fy50M> [Consulté le 21 juin 2016].

DeLEuZE, Gilles (1983). L’image-mouvement, Cinéma 1. Paris : Minuit.

Garrel, Philippe (2014). Rétrospective avec Philippe Azoury, cinéma Nimas. Lisbonne : Lisbon and Estoril Film Festival 2014. $<$ https://www.youtube.com/watch?v=UXa5ik2C2Es $>$ [Consulté le 21 juin 2016].

Rimbaud, Arthur (2001). Poèmes et textes mis en images par Gabriel Lefebvre. Tournai : La Renaissance du Livre.

Thouvenel, Éric (2010). Les images de l'eau dans le cinéma français des années 20. Rennes : Presses universitaires de Rennes. 